Meta

Journal des traducteurs

Translators' Journal

\title{
Les propriétés syntaxiques de l'unité lexicale
}

\section{Alan J. Ford}

Volume 18, numéro 1-2, mars 1973

Actes du deuxième colloque international de linguistique et de traduction. Montréal, 4-7 octobre 1972

URI : https://id.erudit.org/iderudit/002757ar

DOI : https://doi.org/10.7202/002757ar

Aller au sommaire du numéro

Éditeur(s)

Les Presses de l'Université de Montréal

ISSN

0026-0452 (imprimé)

1492-1421 (numérique)

Découvrir la revue

Citer cet article

Ford, A. J. (1973). Les propriétés syntaxiques de l'unité lexicale. Meta, 18(1-2),

47-52. https://doi.org/10.7202/002757ar d'utilisation que vous pouvez consulter en ligne.

https://apropos.erudit.org/fr/usagers/politique-dutilisation/ 


\section{Les propriétés syntaxiques de l'unité lexicale}

Au cours de cette brève communication sur les propriétés syntaxiques de l'unité lexicale, j'utiliserai ce dernier terme de façon presque synonyme du signe linguistique saussurien, c'est-à-dire comme l'association arbitraire d'un signifié, qu'on pourrait considérer comme composé d'une matrice de traits sémantiques ou, si l'on préfère, d'un ensemble fini de postulats de sens, et d'un signifiant, composé d'une matrice de traits phonétiques. La seule différence entre cette unité et le signe saussurien, en dehors de la formulation spécifique de la nature interne du signifié et du signifiant, étant qu'en plus d'une matrice de traits phonétiques, qui correspondrait à l'image acoustique saussurienne, on ajouterait à la structure interne du signifiant un certain nombre de propriétés combinatoires que j'appellerai ici les propriétés syntaxiques.

On pourra constater la nature et l'importance de ces propriétés en considérant un exemple. Prenons la phrase :

(1) L'atelier ferme le dimanche.

Si la langue n'était qu'une liste de formes phonétiques associées à autant de concepts, l'interprétation que l'on donnerait à cette phrase serait nécessairement ambiguë, parce qu'en français il y a au moins quatre concepts pouvant être rattachés à la seule forme ferme, soit :

(i ) ferme $=$ «exploitation agricole »

(ii) ferme $=$ « qui se tient $»$

(iii) ferme $=$ «avec force $»$

(iv) ferme $=$ "causer... ne plus être ouvert »

Mais, dans des circonstances normales, un seul de ces concepts, le quatrième, serait retenu dans l'interprétation de (1). La conclusion que je tire de ce fait est que la seule forme phonétique ou graphique ne recouvre pas l'ensemble du signifiant et que si l'on peut reconnaître, chaque fois, dans (1) qu'il est question du concept (iv), il existe d'autres aspects du signifiant qui nous permettent de le déceler sans risque de méprise. Ce sont ces autres aspects du signifiant que je nomme «propriétés syntaxiques».

Dans le passé, les lexicologues et lexicographes ont été bien conscients de l'importance des propriétés syntaxiques dans la description du signifiant. Dans la plupart des dictionnaires de la langue française on peut donc retrouver ces quatre 
sens de la graphie ferme, signalés par des entrées ou sous-entrées, chacune distinguée des autres par son appartenance à une classe syntaxique ou catégorie différente. Ainsi, (i) : nom, (ii) : adj., (iii) : adv., (iv) : verbe. Mais ce qui ne peut manquer de frapper le linguiste, c'est que la classification syntaxique des dictionnaires a toujours continué d'être celle des huit catégories ou parties du discours mises en relief par les Grecs. Que cet état de choses ait persisté jusqu'à la révolution saussurienne, et même quelque temps après, n'étonne personne puisque le progrès dans le domaine de la syntaxe est resté minime pendant près de deux mille ans. Mais depuis que la linguistique a été affectée par les écrits de Jespersen, Bloomfield, Harris, Martinet et Chomsky, pour ne mentionner que les auteurs les plus importants, il semble que la situation ait considérablement changé et qu'on ne cesse de faire des progrès sensibles dans l'analyse et la documentation des structures syntaxiques de langues comme le français et l'anglais. Il est donc urgent que la lexicologie et la lexicographie commencent à tenir compte des travaux réalisés par les syntacticiens et qu'on puisse éliminer des dictionnaires des parties $\mathrm{du}$ discours ne correspondant à aucune classification scientifique des véritables structures syntaxiques de la langue, pour les remplacer par des propriétés syntaxiques qu'un usager sera capable de relier à une véritable grammaire de sa langue.

Il n'est nul besoin d'illustrer ici les contradictions aberrantes qui résultent de l'application aveugle des catégories de Denys de Thrace à la langue française. Le mélange de critères formels et fonctionnels qui a motivé Platon, Aristote et d'autres dans leur définition des catégories montre suffisamment que cette classification est loin d'être homogène et qu'un verbe, défini essentiellement en termes de sa fonction prédicative par rapport au sujet, ne peut appartenir au même système de classification que l'adjectif, dont le trait distinctif est d'ordre formel, à savoir le fait qu'ils s'accorde avec le nom qu'il modifie. Ceci surtout quand on pense que même en grec la même propriété fonctionnelle qui sert à distinguer le verbe est aussi caractéristique d'un grand nombre de formes graphiques tombant dans la classe des adjectifs.

C'est cette multiplicité de critères qui est, en grande partie, responsable de l'inadéquation des catégories traditionnelles; mais il faut citer le rattachement de ces classes supposément mutuellement exclusives à une seule forme phonétique ou graphique, sans tenir compte des exigences contextuelles nécessaires pour la détermination d'une classe syntaxique. Cette déficience des parties du discours est à la racine de l'embarras du maître d'école qui, dans l'analyse de phrases comme (2) et (3),

(2) Il est venu une semaine

(3) Une semaine vaut mieux que rien

pense se contredire en affirmant qu'une semaine est un syntagme nominal dans (3), mais un adverbe de temps dans (2).

Les techniques mises en relief par l'analyse distributionnelle suffisent à régler ce genre de dilemme. Il est facile de démontrer, par substitution, que la classe d'éléments capables de remplacer une semaine dans (2) tout en formant une expression grammaticale, comme 
(2a) hier, pendant mon absence, tout de suite, etc. ne correspond aucunement à la classe d'éléments capable de remplacer la même forme dans (3), laquelle comprendrait

(3a) Louise, une sieste, du pain sec, etc.

Deux faits ressortent immédiatement de cette classification systématique par commutation :

A. D'abord, que le rattachement d'une forme phonétique ou graphique à une classe syntaxique unique constitue une grosse erreur dans la plupart des cas. Cependant, tous les dictionnaires de la langue française que j'ai pu consulter n'hésitent pas à rattacher semaine, vendredi matin, etc., à la classe "nom », sans aucun commentaire ou explication.

D'ailleurs, on pourrait en dire autant de la plupart des formes qui sont ambiguës du point de vue de leur classification syntaxique. Bleu et vieux, par exemple, sont marqués tous les deux à la fois «nom» et «adjectif », mais un contexte comme (4)

(4) Je n'aime pas $\left\{\begin{array}{l}\text { le bleu } \\ \text { le vieux }\end{array}\right.$

sert à montrer que l'usage nominal de bleu recouvre deux structures syntaxiques différentes, nominalisation et pronominalisation, tandis que la forme vieux correspond uniquement à une pronominalisation, la nominalisation étant associée à la forme vieillesse.

B. Le deuxième fait souligné par une analyse formelle concerne l'impossibilité d'une exclusivité mutuelle de deux classes syntaxiques de signes si l'on se limite à une définition en termes de forme phonétique ou graphique seulement. Une telle classification doit nécessairement admettre que les unités comme vendredi, une semaine, etc., peuvent appartenir autant à la classe nominale $\mathrm{SN}$ qu'à la classe adverbiale ADV. Une telle situation se vérifie jusqu'à un certain point, mais décrite de cette façon elle perd une généralisation linguistique très importante, celle que, entre vendredi (SN) et vendredi (ADV), il existe un rapport de distribution complémentaire. On devrait donc s'attendre à ce qu'un dictionnaire nous dise exactement quand vendredi est « adverbe » et quand il est «nom ». Ou mieux encore, qu'il renvoie à une grammaire qui définit formellement ces classes.

Considérons d'autres observations à partir de la phrase (1). Une analyse distributionnelle permet d'y reconnaître les classes suivantes et de leur fournir la structure que nous lui attribuons dans la figure du numéro (1a)

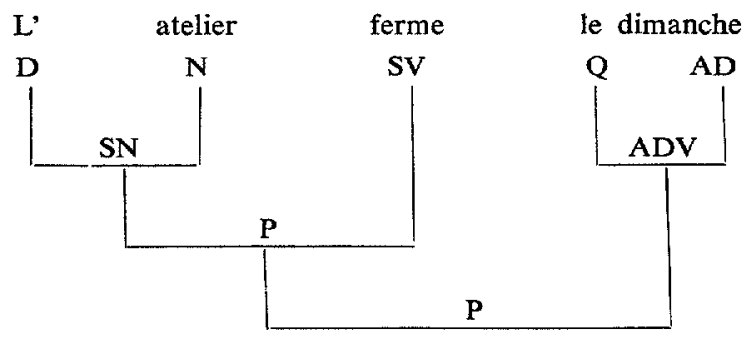


On y distingue les classes suivantes :

$D \quad$ L' / cet, mon, leur, etc.

$N \quad$ atelier / établissement, épicerie, etc.

SN L'atelier / le magasin, la plus grande pharmacie au monde, etc.

$S V$ ferme / est ouvert, est toujours plein, se réunit, etc.

$Q \quad$ le / tous les $-\mathrm{s}$, chaque, certains, etc.

$A D$ le dimanche / dimanche, souvent, quand il n'y a personne, etc.

$P \quad$ L'atelier ferme / La police est en état d'alerte

L'atelier ferme le dimanche, etc.

où / signifie, selon les conventions de l'analyse distributionnelle, « peut être remplacé par », sans que la phrase devienne agrammaticale.

Mais même si la syntaxe distributionnelle évite un grand nombre des problèmes soulevés par les contradictions, lacunes et ambiguités de l'analyse catégorielle de la grammaire traditionnelle, elle est loin de fournir des analyses adéquates. En particulier, elle n'écarte pas la possibilité d'analyses ambiguës, ne répondant pas à notre connaissance intuitive de la structure véritable de la phrase et, à remarquer, de liens avec d'autres phrases. Ce fut l'un des grands apports de la linguistique chomskienne d'établir que les rapports transformationnels pouvaient être utilisés pour éliminer ce genre d'ambiguïté. Examinons de nouveau notre énoncé (1). Outre l'analyse (1a) que nous avons fournie plus haut, la commutation distributionnelle nous permet au moins un second type d'analyse, que nous représentons en (1b) :

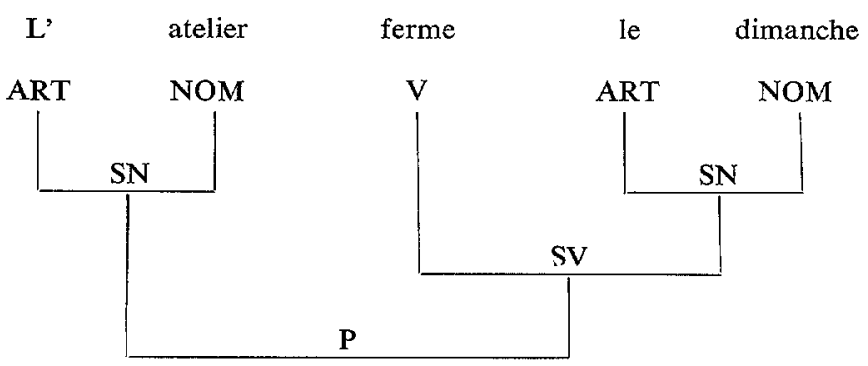

Analyse parallèle à celle qu'on donnerait à une phrase comme (5) :

(5) L'atelier choisit le dimanche.

Comment déterminer laquelle de ces deux analyses est correcte? Le test proposé par une grammaire transformationnelle serait celui de la passivation. Si l'analyse (1b) est la bonne et que ferme appartient à la classe «verbe transitif 》, alors l'application d'une opération de passivation doit fournir une phrase grammaticale. Comme ce n'est pas le cas et que la phrase (1b') est agrammaticale,

(1b') * Le dimanche est fermé par l'atelier

l'analyse (1b) est rejetée en faveur de l'analyse (1a) qui elle-même peut être confirmée par une autre opération transformationnelle; en effet, si le dimanche est un véritable adverbe, il doit pouvoir subir la transformation $d$ ' « antéposition de l'adverbe » avec, comme résultat, 
(1a') Le dimanche l'atelier ferme qui est bien une phrase grammaticale.

Le type de fait que nous venons de signaler n'est qu'un échantillon du travail réalisé en syntaxe depuis déjà plusieurs années, échantillon qui, on le sait, pourrait être multiplié presque indéfiniment. Mais même ces quelques exemples suffisent à montrer qu'il existe un besoin criant de repenser la nature et l'ensemble des renseignements syntaxiques consignés au dictionnaire, et la nécessité, pour tout dictionnaire, de prendre comme base et point de départ ou de référence une grammaire ou théorie d'analyse syntaxique. Une lexicologie ou lexicographie qui se voudrait indépendante de l'analyse syntaxique ne saurait être considérée comme une tentative sérieuse de classification et de définition des unités lexicales d'une langue, sa représentation du signifiant ne rendant nullement compte des faits.

Évidemment, il importera au lexicologue-lexicographe de déterminer le moyen d'évaluer et de formuler les données fournies par diverses analyses syntaxiques. À mon avis, cela dépendra essentiellement de la nature et de la fonction du dictionnaire. Un exemple peut encore servir à illustrer le sens de cette remarque.

Dans l'analyse (1a) de notre énoncé (1), nous avons signalé comment une analyse des structures, même au niveau de surface, détermine l'occurrence de le devant dimanche comme indice d'appartenance à une classe que nous avons appelée «quantificateur », et ceci à cause de son contexte syntaxique, qui peut être résumé sous la forme

$$
\mathrm{P}-\mathrm{ADV} \text {. }
$$

De plus, à cause de la synonymie de (1) avec la phrase (6),

(6) L'atelier ferme tous les dimanches

le signifié de le dans ce contexte semble synonyme avec celui normalement associé à tout, c'est-à-dire celui qu'on représente souvent par le symbole logique $\forall$, ou "quantificateur universel ». On doit se demander sous quelle forme ce type de fait peut être explicité dans un dictionnaire. Ainsi, serait-il nécessaire de l'exprimer dans une sous-entrée spéciale de la forme graphique le? C'est là l'approche adoptée, notamment, par le Petit Robert qui se félicite, avec raison je pense, d'avoir tenu compte des divers emplois de cette forme. Mais les inconvénients de cette option ne manquent pas. L'article se rapportant à le dans le Petit Robert est long à lire, difficile à interpréter (les termes du métalangage, en particulier les noms des classes syntaxiques, ne renvoient à aucune grammaire de la langue française) et ne remplit pas sa fonction lexicologique, ne nous transmet pas la connaissance des signifiés de le, puisqu'il se limite à l'énumération de ses différents signifiants. Il est instructif, à ce sujet, de comparer la forme de cette rubrique avec l'affirmation que fait $\mathrm{M}$. Rey dans la préface du dictionnaire : "Le rôle essentiel d'un dictionnaire de langue consiste pour chaque mot, ou plus exactement pour chaque signifiant, à répartir de manière exacte et claire les différents aspects du signifié. » Il apparaît donc des divergences entre le but qu'on s'était proposé et le résultat obtenu, écart particulièrement frappant dans le cas de le. L'usager ayant consulté le Petit Robert à cette rubrique, quelle réponse est-il en mesure de donner à la 
question : «Que signifie le?» Pour la plupart des signifiants qui comportent cette forme graphique, aucune réponse n'est évidemment possible. S'agirait-il alors d'une lacune du dictionnaire? Dans un sens, non, puisqu'il n'existe souvent aucun signifié directement associé à l'utilisation de la forme le, même munie d'un contexte syntaxique. Demander le sens de le dans la plupart de ses contextes syntaxiques serait équivalent à demander la signification du fait que, dans la phrase

(7) Albert pleure

pleure suit Albert, ou Albert précède pleure. Or, ce fait n'a aucun signifié, il exprime une servitude ou règle grammaticale du français. De la même façon, la présence de le dans la plupart de ses contextes est déterminée par les règles syntaxiques du français ; il n'y a aucun signifié direct à rattacher à ce phénomène. Si l'on peut adresser une critique à la rubrique le du Petit Robert, c'est qu'on essaie d'y présenter le travail d'une grammaire en même temps que celui d'un dictionnaire. Selon la définition de M. Rey, la plupart des entrées pour le ne devraient pas se trouver au dictionnaire. Ou alors, si l'on désire être systématique, on devrait inclure d'autres éléments provenant des règles syntaxiques, comme les terminaisons -ion, $-r$, -ant, les formes que, est, entre autres, sans parler des opérations de permutation où elles jouent.

Il reste évidemment les quelques cas de le auxquels on peut rattacher un signifié dans un contexte syntaxique déterminé, tel notre énoncé (1). Il ressort clairement qu'on rend mieux compte des diverses associations de cette forme en les rattachant individuellement aux quelques éléments lexicaux avec lesquels ils se trouvent en combinaison. Ainsi, la rubrique de dimanche expliciterait l'effet de la présence de la forme le sur le signifiant, si cette présence menait à un changement de sens.

Remarquons que, de ce point de vue, la notion de «transformation » sert à définir le concept d'unité lexicale. Toute forme phonétique présente explicitement dans une règle de transformation n'a aucun besoin de paraître au dictionnaire. En tant que telle, elle forme une classe syntaxique unique dont la valeur sémantique est fournie exclusivement en termes de l'opération transformationnelle. Une telle façon de procéder sert à expliciter, dans une description linguistique (c'est-àdire une grammaire en conjonction avec un dictionnaire), une distinction déjà établie par beaucoup de linguistes, dont Martinet par l'opposition entre monèmes grammaticaux et monèmes lexicaux, et par d'autres écoles structuralistes, qui opposent lexème à grammème.

AlAN J. FORD 